\title{
Assessment of diabetic teleretinal imaging program at the Portland Department of Veterans Affairs Medical Center
}

\author{
Grace L. Tsan, OD; ${ }^{1 *}$ Keely L. Hoban, OD; ${ }^{1}$ Weon Jun, OD, FAAO; ${ }^{1-2}$ Kevin J. Riedel, OD; ${ }^{1}$ Amy L. Pedersen, \\ OD; ${ }^{1}$ John Hayes, PhD $^{2}$ \\ ${ }^{1}$ Portland Department of Veterans Affairs Medical Center, Portland, OR; ${ }^{2}$ College of Optometry, Pacific University \\ Oregon, Forest Grove, OR
}

\begin{abstract}
We conducted a retrospective chart review of 200 diabetic patients who had teleretinal imaging performed between January 1, 2010, and January 1, 2011, at Portland Department of Veterans Affairs (VA) Medical Center outpatient clinics to assess the effectiveness of the diabetic teleretinal imaging program. Twenty patients (10\%) had diabetic retinopathy. Ninety percent of the available teleretinal imaging studies were of adequate quality for interpretation. In accordance with local VA policy at that time, all teleretinal imaging patients should have been referred for a dilated retinal examination the following year. Image readers referred $97.5 \%$ of the patients to eye clinics for subsequent eye examinations, but the imagers scheduled appointments for only $80 \%$ of these patients. The redundancy rate, i.e., patients who had an eye examination within the past 6 mo, was $11 \%$; the duplicate recall rate, i.e., patients who had a second teleretinal imaging performed within $1 \mathrm{yr}$ of the eye examination, was $37 \%$. Rates of timely diabetic eye examinations at clinics with teleretinal imaging programs, particularly when teleretinal imaging and eye clinics were colocated at the same community-based outpatient clinic, were higher than at those without a teleretinal imaging program. We concluded that the Portland VA Medical Center's teleretinal imaging program was successful in increasing the screening rate for diabetic retinopathy.
\end{abstract}

Key words: diabetes mellitus, diabetic retinal examination, diabetic retinopathy, dilated eye examination, outpatient clinic, primary care, screening, telehealth, teleretinal imaging, teleretinal imaging program.

\section{INTRODUCTION}

Diabetes mellitus affected 25.8 million (8.3\%) Americans in 2011 [1]. It affected 10.9 million (26.9\%) of the 40.5 million Americans 65 yr or older. Thus, diabetes is prevalent among the predominantly elderly Veteran patient population [1-2]. Diabetic retinopathy (DR) is the leading cause of blindness in working-age adults [3]. It affects 78 percent of those with diabetes for 15 or more years [4]. Early diagnosis and treatment of DR is important, because treatment is 90 percent effective in preventing blindness [3]. However, fewer than 50 percent of American

\footnotetext{
Abbreviations: $\mathrm{AMD}=$ age-related macular degeneration, $\mathrm{BMI}=$ body mass index, $\mathrm{CBOC}=$ community-based outpatient clinic, CPRS $=$ Computerized Patient Record System, DR = diabetic retinopathy, EPRP $=$ External Peer Review Program, HbA1C = hemoglobin A1C, HDL = high density lipoprotein, HEDIS = Healthcare Effectiveness Data and Information Set, LDL = low density lipoprotein, NPDR = nonproliferative diabetic retinopathy, PVAMC = Portland Department of Veterans Affairs Medical Center, VA = Department of Veterans Affairs, VHA = Veterans Health Administration, VistA = Veteran Health Information System and Technology Architecture.

*Address all correspondence to Grace L. Tsan, OD; Raymond G. Murphy VA Medical Center, 1501 San Pedro SE, Albuquerque, NM 87108; 505-265-1711, ext 4229; fax: 505256-5761. Email: grace.tsan@va.gov

http://dx.doi.org/10.1682/JRRD.2014.03.0077
} 
diabetic patients receive annual DR screening examinations [5-6].

Nonmydriatic digital retinal imaging with remote image interpretation (teleretinal imaging) is an emerging healthcare technology for screening patients for DR [78]. This technology allows rapid retinal imaging without dilation of the pupil in primary care clinical offices. It has been shown to have excellent diagnostic precision for DR compared with examinations with dilated pupils in eye care providers' offices (dilated eye examination) and improves the rate of DR screening [9-11].

In 1999, the Department of Veterans Affairs' (VA's) Veterans Health Administration (VHA) collaborated with the Joslin Diabetes Center in Boston, Massachusetts, to implement a pilot teleretinal imaging program. It involved a nonmydriatic digital retinal imaging platform; imaging stations were installed at VA medical centers and community-based outpatient clinics (CBOCs) in New England and the Pacific Northwest. Images captured at remote imaging stations were transmitted to reading centers at the Joslin Diabetes Center and the VA Puget Sound Healthcare System in Seattle, Washington. This pilot program proved to be highly successful in identifying patients in need of timely further care for DR while at the same time recommending eye care at appropriate intervals for those with little or no risk factors for retinopathy progression [7].

In 2001, VHA convened an expert panel to address issues of clinical application, quality and training, information technology, and healthcare infrastructure needs for deploying such a teleretinal imaging program. VHA deployed teleretinal imaging as a systemwide screening method for DR in 2006 [7-8].

VHA has also established an External Peer Review Program (EPRP) to provide medical centers and outpatient clinics with diagnosis and procedure-specific quality of care information [9]. The EPRP program provides a database for both internal and external comparison of clinical care. VA uses the Healthcare Effectiveness Data and Information Set (HEDIS) [10] to continuously improve the EPRP clinical indicator for evaluation of diabetic patients for DR. Veteran patients will meet the HEDIS DR screening performance criteria by evidence of diabetic eye examinations by a VA or non-VA eye care provider (optometrist or ophthalmologist) or adequate teleretinal imaging by a certified teleretinal imager with reading by a certified teleretinal image reader [9].
We conducted a randomized retrospective cohort study to determine the effectiveness of the current Portland VA Medical Center's (PVAMC's) teleretinal imaging program as a screening method in detecting DR and subsequent follow-up of the patients. Previous DR screening studies have demonstrated that the teleretinal imaging program improves DR assessment rates and increases enrollment of diabetic patients in eye clinics [11-13]. However, none of these studies assessed the effectiveness of the program when diabetic patients were enrolled in both an eye clinic and a teleretinal imaging program. In addition, we also analyzed the association of DR with various risk factors and comorbid conditions. The results of this study formed the basis of the current report.

\section{METHODS}

The PVAMC operated a total of eight outpatient clinics, including five teleretinal imaging screening programs at CBOCs in Salem, West Linn, Bend, and Hillsboro, Oregon; and Vancouver, Washington.

For the teleretinal imaging screening, four nonmydriatic photographs were obtained (three of the retina and one of the external ocular structures) with a Topcon TRC-NW6S digital retinal camera (Topcon Medical Systems; Oakland, New Jersey). These photographs were then transferred to the VA Computerized Patient Record System (CPRS), where they were analyzed by a certified teleretinal image reader, who was either a licensed optometrist or ophthalmologist. Based on the interpretation of these images, the reader assessed whether (1) the image quality was adequate for interpretation; (2) any DR or clinically significant macular edema was present and if so, at what level; and (3) there were any comorbid nondiabetic ocular conditions. The reader would then determine the recommended recall to the eye clinic, which was transferred back to the imagers for scheduling. At PVAMC, imagers were certified teleretinal imaging technicians who were responsible for identifying and scheduling patients for teleretinal imaging as well as following up on readers' recommendations, including scheduling patients for eye clinic appointments [14]. It was PVAMC policy in 2010 and 2011 to refer all teleretinal imaging patients to the eye clinic for dilated eye examinations in the following year regardless of whether or not they were found to have DR. 
We reviewed the CPRS records of a random sample of 200 diabetic patients who had teleretinal imaging performed between January 1, 2010, and January 1, 2011, at the PVAMC CBOCs in Salem, West Linn, Bend, and Hillsboro, Oregon; and Vancouver, Washington. Data collected were demographic information including age, sex, and race; risk factors including blood glucose, hemoglobin A1C (HbA1C), blood pressure, body mass index (BMI), serum lipid levels (total cholesterol, triglyceride, high density lipoprotein [HDL] cholesterol, and low density lipoprotein [LDL] cholesterol); quality of retinal imaging; stages of DR and comorbid eye conditions such as glaucoma and age-related macular degeneration (AMD); duration of diabetes; and effectiveness of subsequent follow up after teleretinal imaging.

When patients had a dilated eye examination performed within $6 \mathrm{mo}$ at VA or elsewhere before teleretinal imaging, the teleretinal imaging was considered to be redundant, i.e., unnecessary. Likewise, when patients had a pending teleretinal imaging recall in addition to a pending scheduled eye clinic examination appointment within $1 \mathrm{yr}$, the teleretinal imaging recall was considered to be a duplicate recall. The percentage redundancy rate was calculated by dividing the number of patients with a redundant teleretinal imaging by the total number of the study population, multiplied by 100 . Likewise, the percentage duplicate recall rate was calculated by dividing the number of patients with duplicate teleretinal imaging before the dilated eye examination by the total number of study population, multiplied by 100 .

This study was approved by the PVAMC Institutional Review Board prior to the initiation of the study. In addition, data from the Veteran Health Information System and Technology Architecture (VistA) on June 2, 2010, were obtained by running an ad hoc report to determine the rates of timely diabetic retinal examinations, i.e., retinal examinations performed annually or biennially (i.e., every 2 yr) by a VA or non-VA eye care provider depending on whether there were diabetic eye pathologies or adequate teleretinal imaging by a certified teleretinal imager with reading by a certified teleretinal image reader, at all eight PVAMC outpatient clinics (Salem, Hillsboro, Vancouver, Bend, Portland, East Portland, North Coast, and West Lynn). The report showed the number of PVAMC diabetic patients meeting the HEDIS DR screening performance measure. Similar data were obtained for Hillsboro and West Lynn clinics from January 2010 through January 2012, because these two clinics established teleretinal imaging programs during this time period on December 8, 2010, and November 28, 2011, respectively.

Logistic regression [15] was used to determine the association of various systemic risk factors and comorbid eye conditions with DR. For purposes of the logistic regression, we combined various levels of retinopathy and defined patients as either having some level of retinopathy or no retinopathy. The chi-square test was used to determine the difference in distribution between two groups for dichotomous variables, and the $t$-test was used for continuous variables [15].

\section{RESULTS}

\section{Patient Population}

This study included 200 patients, randomly selected from a total of 1,567 diabetic patients, who had teleretinal imaging performed between January 1, 2010, and January 1, 2011. Their mean age was $64.7 \mathrm{yr}$ (standard deviation = $10.2 \mathrm{yr}) ; 194$ (97\%) were male and 192 (91\%) were Caucasian. Table 1 shows the number of patients with DR. Of the 20 patients (10\%) who were found to have DR, 11 had mild nonproliferative DR (NPDR), 6 had moderate NPDR, 1 had severe NPDR, and 2 had proliferative DR.

\section{Risk Factors and Comorbid Conditions}

Table 2 summarizes risk factors associated with diabetes and comorbid eye conditions. There was a significant association between developing DR and years with diabetes mellitus (Table 3, $p=0.002$ ). The model predicted that 50 percent of the population would develop DR after having diabetes for 34 yr. The relationship

Table 1.

Prevalence of patients with diabetic retinopathy (DR) in Computerized Patient Record System $(n=200)$.

\begin{tabular}{lc}
\hline Diagnosis & No. (\%) of Patients \\
\hline DR & $20(10.0)$ \\
Mild NPDR & $11(5.5)$ \\
Moderate NPDR & $6(3.0)$ \\
Severe NPDR & $1(0.5)$ \\
Proliferative DR & $2(1.0)$ \\
\hline
\end{tabular}

NPDR = nonproliferative DR. 
Table 2.

Participant risk factors and comorbid conditions.

\begin{tabular}{|c|c|c|c|c|}
\hline \multirow{2}{*}{ Factor/Condition } & \multicolumn{3}{|c|}{ Mean \pm SD $^{*}$} & \multirow{2}{*}{$p$-Value } \\
\hline & Overall & With No DR & With DR & \\
\hline Body Mass Index $(n=178)$ & $33.8 \pm 7.2$ & $33.9 \pm 7.4$ & $34.2 \pm 6.4$ & 0.89 \\
\hline Systolic Blood Pressure (mm Hg) $(n=199)$ & $130.1 \pm 15.4$ & $130.4 \pm 15.9$ & $130.9 \pm 14.9$ & 0.90 \\
\hline Diastolic Blood Pressure (mm Hg) $(n=199)$ & $75.0 \pm 11.1$ & $75.2 \pm 10.7$ & $76.0 \pm 15.4$ & 0.77 \\
\hline Fasting Blood Glucose (mg/dL) $(n=197)$ & $154.7 \pm 58.0$ & $154.5 \pm 60.5$ & $176.0 \pm 38.9$ & 0.04 \\
\hline Fasting Hemoglobin A1C (\%) $(n=197)$ & $7.3 \pm 1.5$ & $7.3 \pm 1.5$ & $7.9 \pm 1.4$ & 0.05 \\
\hline Fasting High Density Lipoprotein Cholesterol (mg/dL) $(n=152)$ & $43.0 \pm 13.7$ & $42.9 \pm 14.0$ & $45.1 \pm 12.5$ & 0.59 \\
\hline Fasting Low Density Lipoprotein Cholesterol (mg/dL) $(n=139)$ & $88.6 \pm 36.0$ & $88.8 \pm 36.5$ & $73.5 \pm 26.3$ & 0.16 \\
\hline Diabetes Duration (yr) $(n=199)$ & $8.6 \pm 6.7$ & $8.1 \pm 6.1$ & $13.1 \pm 9.3$ & 0.002 \\
\hline Current Tobacco User $(\%)(n=199)$ & 99.0 & 99.4 & 100.0 & 0.73 \\
\hline Past Vascular Surgery (\%) $(n=199)$ & 3.3 & 3.2 & 5.0 & 0.67 \\
\hline Age-Related Macular Degeneration (\%) $(n=196)$ & 4.0 & 4.4 & 0.0 & 0.35 \\
\hline Clinically Significant Macular Edema $(\%)(n=180)$ & 2.2 & 0.0 & 20.0 & $<0.001$ \\
\hline
\end{tabular}

Table 3.

Logistic regression models predicting diabetic retinopathy.

\begin{tabular}{|c|c|c|c|c|c|}
\hline Variable & B & SE & Chi-Square & $p$-Value & Odds Ratio \\
\hline
\end{tabular}

between DR and HbA1C approached statistical significance $(p=0.05)$, with the model predicting 50 percent DR with an HbA1C of 15.9 percent. Regression analysis revealed that age, blood pressure, sleep apnea, weight, BMI, total cholesterol, triglyceride, HDL cholesterol, and LDL cholesterol were not associated with DR. The comorbid conditions of AMD and glaucoma were also not associated with DR.

\section{Teleretinal Imaging and Subsequent Patient Follow-Up}

Of the 195 teleretinal images available for evaluation, 175 (89.7\%) were judged to be of adequate quality for interpretation and 20 (10.3\%) were judged to be of poor quality.

Twenty-two patients had had a dilated eye examination within 6 mo prior to being imaged. Since these patients had already had a recent eye examination within the past 6 mo, there was no need for the teleretinal imaging. Thus, there was a redundancy rate of 11 percent.
Seventy-four patients had a second teleretinal imaging performed within $1 \mathrm{yr}$ of the dilated eye examination. This represented a duplicate recall rate of 37 percent.

Image readers referred 97.5 percent of the patients (i.e., 195 of 200 patients) to the eye clinics for subsequent eye examinations, but the imagers at the primary care clinics scheduled appointments for only 80 percent (i.e., 156 of 195) of these patients. According to local VA facility policy at that time, all patients should have been referred to an eye clinic for dilated retinal examinations the following year.

\section{Effect of Teleretinal Imaging Program on Rate of Timely Diabetic Retinal Examinations}

To determine the potential effect of teleretinal imaging screening programs on the rate of timely diabetic retinal examinations, we obtained data from all eight outpatient clinics with and without teleretinal imaging programs. As of June 2, 2010, the PVAMC had three 
CBOCs with teleretinal imaging programs, i.e., Bend, Salem, and Vancouver. As shown in Figure 1, these three clinics had higher rates of timely diabetic retinal examinations, particularly when the teleretinal imaging program and eye clinic were colocated at the same CBOC, than the other five CBOCs without teleretinal imaging programs. Between January 2010 and January 2012, two outpatient clinics established teleretinal imaging screening programs. As shown in Figure 2, the rates of timely diabetic retinal examinations increased from 70-80 percent to more than 90 percent within a few months.

\section{DISCUSSION}

The data presented in this study demonstrate that teleretinal imaging programs appear to be an efficient method to triage patients in primary care clinics for further evaluation with an eye care provider. Approximately 90 percent of the available teleretinal images were of adequate quality for reader interpretation. Twenty patients $(10 \%)$ had some degree of DR. It was policy at the time of this study that all patients should be referred to the eye clinic for dilated retinal examinations the following year. Image readers referred 97.5 percent of the patients (195 of 200 patients) to the eye clinics for subsequent eye examinations. However, the imagers at the pri-

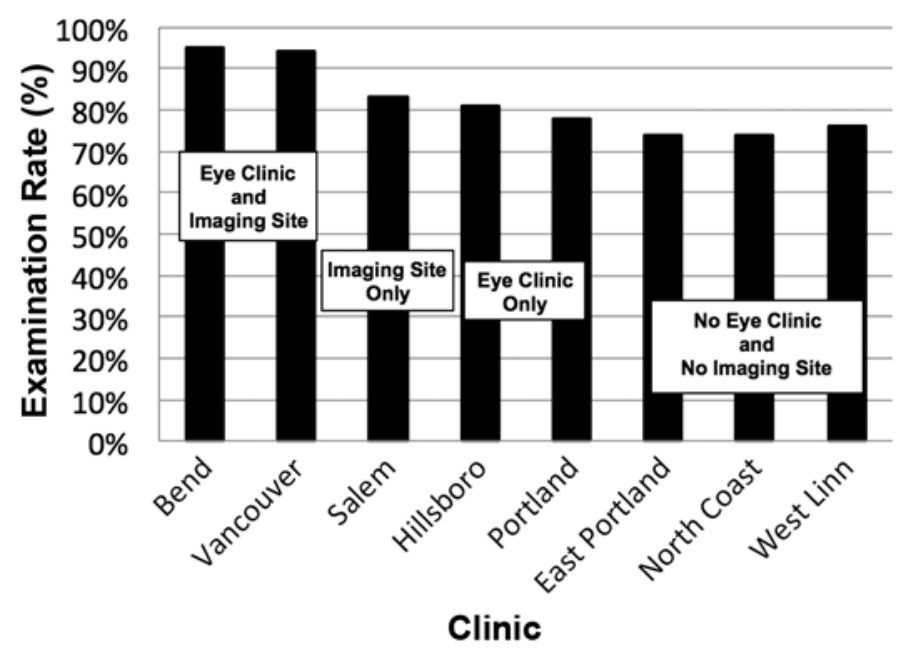

Figure 1.

Rates of timely diabetic retinal examination at the Portland Department of Veterans Affairs Medical Center primary care clinics (Veteran Health Information System and Technology Architecture Clinical Reminder snapshot on June 2, 2010). mary care clinics scheduled appointments for only 80 percent (156 of 195) of these patients. The redundancy rate was 11 percent (22 patients), and the duplicate recall rate was 37 percent (74 patients).

In addition, the teleretinal imaging program appeared to improve the screening rate for diabetic eye diseases as demonstrated by an increased rate of timely diabetic retinal examinations when a teleretinal imaging program was established in the primary care clinic and that CBOCs with teleretinal imaging programs, particularly where a teleretinal imaging program and eye clinic were colocated at the same CBOC, appeared to perform better in their rates of timely diabetic retinal examinations than those clinics without such programs. This latter finding is not surprising, as colocation of the teleretinal imaging program and eye clinic in the same CBOC promotes the routine use of teleretinal imaging as a DR screening method.

At PVAMC, the target diabetic patients for teleretinal imaging are those who have not had an eye examination within the past year; those who failed to keep their eye clinic appointment or who failed to provide documentation of an eye examination by an eye care provider (optometrist or ophthalmologist); those with poorly controlled blood glucose on insulin or a HbA1C $>8$ percent but with no DR; and those with poorly controlled blood pressure, exceeding a systolic measurement of $140 \mathrm{~mm}$ $\mathrm{Hg}$ and a diastolic measurement of $90 \mathrm{~mm} \mathrm{Hg}[6-7,14]$.

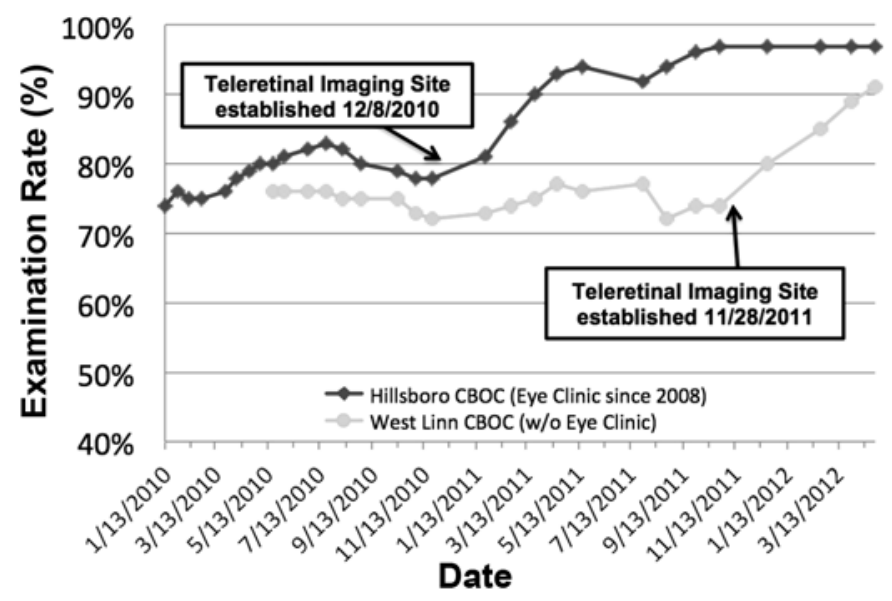

Figure 2.

Changes in rates of timely diabetic retinal examination after implementation of teleretinal imaging program. $\mathrm{CBOC}=$ communitybased outpatient clinic. 
On the other hand, patients who had poorly controlled diabetes with a history of DR or laser procedures, other eye diseases, pregnancy, or high-risk diabetic patients were not candidates for teleretinal screening and would benefit more from eye clinic follow-up [6-7,14]. An additional advantage of teleretinal imaging was the ability for the program to identify patients with previous DR or other eye diseases who had been lost to follow-up.

We also found that the risk for developing DR correlated with the number of years having diabetes mellitus and increased HbA1C levels. Other retinal findings, such as glaucoma and AMD, were observed with teleretinal imaging. While none of these other retinal findings was found to be associated with DR, we were able to identify these asymptomatic patients with risk factors for sightthreatening diseases who might not have otherwise sought eye care.

We were particularly interested in subsequent followup after teleretinal imaging. We found a very high rate of referral; 97.5 percent of the patients (i.e., 195 of 200 patients) were referred by the image readers to eye clinics for further eye examinations. It was the PVAMC policy at that time to refer all teleretinal imaging patients to the eye clinic for dilated eye examinations in the following year whether or not they had DR. However, the imagers only scheduled 80 percent of these patients (i.e., 156 of 195) for subsequent eye clinic appointments. The reasons for this low scheduling rate were not clear. However, this is certainly one area that needs to be addressed.

Additionally, we observed that 11 percent of our patients had recently had an eye examination within 6 mo prior to the teleretinal imaging. Since they had already had an eye examination in the last 6 mo, there was no indication for redundant teleretinal imaging. This represents an inefficient use of healthcare resources. Likewise, 37 percent of the patients had a duplicate teleretinal recall before the scheduled eye examination. Again, the duplicate recall was not necessary and represents poor use of resources with unnecessary visits.

Based on these findings, we identified the following areas for improvement: reducing the redundancy rate by carefully selecting patients for teleretinal imaging to ensure that patients have had no such imaging within the last 6 mo; improving the follow-up eye examination rate by ensuring that all referred patients receive eye clinic appointments; and reducing the duplicate recall rate by avoiding a second teleretinal imaging before an eye clinic dilated eye examination. In addition, we should take advantage of the Clinical Reminder report for diabetic retinal examinations in VistA to identify diabetic patients who are due for their timely diabetic retinal examinations. This ad hoc report identifies any diabetic patients who are due for their timely diabetic retinal examinations based on whether the diabetic retinal examination clinical reminder date is past due in CPRS. The due date is reset automatically in CPRS based on diabetic retinal examination coding or DR International Classification of Diseases, 9th Revision codes; it could also be manually reset by a provider if a diabetic retinal examination was performed by a non-VA eye care provider.

This study has a number of limitations. It was a retrospective cohort study, and the subject sample size was small. Diabetic patients require ongoing, lifelong DR surveillance, and long-term follow-up is critical to evaluate the effectiveness of the teleretinal imaging program. However, our study's duration was limited to only 1 yr. The teleretinal imaging program has been validated in screening for DR; however, its use for identifying other ocular pathologies has not yet been supported. Future studies should examine the effectiveness of teleretinal imaging in identifying other ocular pathologies, such as glaucoma and AMD.

\section{CONCLUSIONS}

We conclude that the PVAMC's teleretinal imaging program successfully targeted mostly patients with low risk for DR and increased the screening rate for diabetic eye diseases. We recommend that VA medical centers implement a teleretinal imaging program at all VA primary care clinics to improve the annual DR screening rate.

\section{ACKNOWLEDGMENTS}

\section{Author Contributions:}

Study design: G. L. Tsan, W. Jun.

Collection, analysis, and interpretation of data: G. L. Tsan, K. L. Hoban, W. Jun, K. J. Riedel, A. L. Pedersen, J. Hayes. Preparation and submission of manuscript: G. L. Tsan. Review and revision of manuscript: K. L. Hoban, W. Jun, K. J. Riedel, A. L. Pedersen, J. Hayes.

Financial Disclosures: The authors have declared that no competing interests exist.

Funding/Support: This material was unfunded at the time of manuscript preparation. 
Additional Contributions: The authors thank Min-Fu Tsan, MD, $\mathrm{PhD}$, Senior Research Scientist at the McGuire Research Institute, Richmond, Virginia, for critical review of the manuscript. Please note the authors' current affiliations: Dr. Tsan, Raymond G. Murphy VA Medical Center, Albuquerque, New Mexico; Dr. Jun, VA Portland Health Care System, Portland, Oregon; Dr. Hoban, Tigard Vision Center, Tigard, Oregon; Dr. Pedersen, Kaiser Permanente Riverside Medical Center, Riverside, California; Dr. Riedel, Oregon Eye Specialists, Tualatin, Oregon; and Dr. Hayes, Pacific University College of Optometry, Forest Grove, Oregon.

Institutional Review: This study was approved by the PVAMC Institutional Review Board with a waiver of the requirement of informed consent prior to the initiation of the study.

Participant Follow-Up: The authors do not plan to inform participants of the publication of this study because contact information is unavailable.

Disclaimers: The views expressed in this article are those of the authors and do not represent an official position of the institutions.

\section{REFERENCES}

1. National Diabetes Information Clearinghouse. National diabetes statistics 2011 [Internet]. Bethesda (MD): National Institute of Diabetes and Digestive and Kidney Diseases; 2011. Available from: http://www.niddk.nih.gov/

2. Miller DR, Safford MM, Pogach LM. Who has diabetes? Best estimates of diabetes prevalence in the Department of Veterans Affairs based on computerized patient data. Diabetes Care. 2004;27(Suppl 2):B10-21. [PMID:15113777] http://dx.doi.org/10.2337/diacare.27.suppl_2.B10

3. American Diabetes Association. Diabetic retinopathy. Diabetes Care. 2000;23(Suppl 1):S73-76. [PMID:12017685]

4. Klein R, Klein BE, Moss SE, Davis MD, DeMets DL. The Wisconsin epidemiologic study of diabetic retinopathy. III. Prevalence and risk of diabetic retinopathy when age at diagnosis is 30 or more years. Arch Ophthalmol. 1984; 102(4):527-32. [PMID:6367725] http://dx.doi.org/10.1001/archopht.1984.01040030405011

5. Ferris FL 3rd, Davis MD, Aiello LM. Treatment of diabetic retinopathy. N Engl J Med. 1999;341(9):667-78.

[PMID:10460819] http://dx.doi.org/10.1056/NEJM199908263410907

6. Garg S, Davis R. Diabetic retinopathy screening update. Clin Diabetes. 2009;27:140-45.

http://dx.doi.org/10.2337/diaclin.27.4.140

7. Conlin PR, Fisch BM, Orcutt JC, Hetrick BJ, Darkins AW. Framework for a national teleretinal imaging program to screen for diabetic retinopathy in Veterans Health Administration patients. J Rehabil Res Dev. 2006;43(6):741-48. [PMID:17310423] http://dx.doi.org/10.1682/JRRD.2005.08.0146

8. Cavallerano AA, Conlin PR. Teleretinal imaging to screen for diabetic retinopathy in the Veterans Health Administration. J Diabetes Sci Technol. 2008;2(1):33-39.

\section{[PMID:19885175]}

http://dx.doi.org/10.1177/193229680800200106

9. Department of Veterans Affairs. External peer review program. VHA Directive 2008-032 [Internet]. Washington (DC): Department of Veterans Affairs; 2008 Jun 23. Available from: http://www1.va.gov/vhapublications

10. National Committee for Quality Assurance. The Healthcare Effectiveness Data and Information Set (HEDIS) and Quality Measurement [Internet]. Washington (DC): National Committee for Quality Assurance. Available from:

http://www.ncqa.org

11. Lin DY, Blumenkranz MS, Brothers RJ, Grosvenor DM. The sensitivity and specificity of single-field nonmydriatic monochromatic digital fundus photography with remote image interpretation for diabetic retinopathy screening: A comparison with ophthalmoscopy and standardized mydriatic color photography. Am J Ophthalmol. 2002;134(2): 204-13. [PMID:12140027] http://dx.doi.org/10.1016/S0002-9394(02)01522-2

12. Farley TF, Mandava N, Prall FR, Carsky C. Accuracy of primary care clinicians in screening for diabetic retinopathy using single-image retinal photography. Ann Fam Med. 2008;6(5):428-34. [PMID:18779547]

http://dx.doi.org/10.1370/afm.857

13. Mansberger SL, Gleitsmann K, Gardiner S, Sheppler C, Demirel S, Wooten K, Becker TM. Comparing the effectiveness of telemedicine and traditional surveillance in providing diabetic retinopathy screening examinations: A randomized controlled trial. Telemed J E Health. 2013; 19(12):942-48. [PMID:24102102] http://dx.doi.org/10.1089/tmj.2012.0313

14. Department of Veterans Affairs. Teleretinal screening program. VA Northwest Health Network policy memorandum 10N20-015. Washington (DC): Department of Veterans Affairs; 2012.

15. Matthews DE, Farewell VT. Using and understanding medical statistics. 2nd ed. Basel (Switzerland): S. Karger AG; 1988.

Submitted for publication April 17, 2014. Accepted in revised form January 6, 2015.

This article and any supplementary material should be cited as follows:

Tsan GL, Hoban KL, Jun W, Riedel KJ, Pedersen AL, Hayes J. Assessment of diabetic teleretinal imaging program at the Portland Department of Veterans Affairs Medical Center. J Rehabil Res Dev. 2015;52(2):193-200. http://dx.doi.org/10.1682/JRRD.2014.03.0077

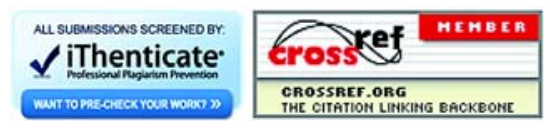


